ODONTOLOGIA SANMARQUINA

ISSN: $1560-9111$

Artículo Original

\section{Conocimiento, aplicación y apreciación de la tecnología de la información y comunicación (TIC) 2.0 de estudiantes y docentes de la Facultad de Odontología de la Universidad Nacional Mayor de San Marcos: Pregrado y posgrado}

Knowledge, application and assessment of the information technology and communication (ICT) 2.0 students and teachers from the Faculty of Dentistry of the University of San Marcos: undergraduate and graduate

\begin{abstract}
Resumen
Objetivo: Se tuvo como objetivo analizar el conocimiento, aplicación y apreciación sobre las tecnologías de la información y comunicación (TIC) de la web 2.0 en el ámbito educativo. Estas consideran el uso de aplicaciones y páginas web dispuestas a utilizar la inteligencia colectiva a través del software social con el fin de facilitar los procesos de aprendizaje. Materiales y método: Se desarrolló un estudio observacional, descriptivo, transversal en una muestra probabilística aleatoria simple de 173 y 109 estudiantes de pregrado y posgrado respectivamente, así como 54 docentes. El cuestionario fue validado por juicio de experto obteniendo un coeficiente V de Aiken igual a 1 y una confiabilidad Alfa de Conbrach de 0.75. Resultados: Los tres grupos estudiados reconocieron a las herramientas de comunicación como un mecanismo de compartir información, mejorar el vínculo docente y desarrollar trabajo colaborativo con porcentajes superiores al $80 \%$. La apreciación sobre el uso de las mismas es calificada como bueno en el $58.9 \%, 66.1 \%$ y $72 \%$ de los estudiantes de pregrado, posgrado y docentes respectivamente. El uso de estas herramientas es mayor en los estudiantes de pregrado, $92.6 \%$, seguido por los de posgrado, $82.6 \%$, y docentes, $69.8 \%$. Existe conocimiento de las distintas herramientas de generación y publicación de contenidos en los tres grupos estudiados. La herramienta de recuperación y búsqueda de información más utilizada por docentes y estudiantes de posgrado fueron las bases de datos especializadas. Conclusión: Existe una tendencia positiva a utilizar estas herramientas dentro del ámbito educativo. Palabras clave: Educación superior, Tecnología educacional, Navegador web, Internet
\end{abstract}

\section{Abstract}

Objective: This research aims to analyze the knowledge, application and assessment about the Information and Communication Technology (ICT) of Web 2.0 in education. The Web 2.0 consider the use of applications and websites willing to use the collective intelligence through social software to facilitate the learning processes. Materials and method: An observational, descriptive, cross-sectional study was conducted in a simple random pro-bability sample of 173 students and 109 undergraduate and graduate respectively and 54 teachers. The questionnaire was validated by expert judgment obtaining a coefficient Aiken V equal to 1 , and Cronbach alpha reliability of 0.75 . Results: The results showed that the three groups recognized communication tools as a mechanism to share information, improve teacher relationship and develop collaborative work with percentages above 80 $\%$. The assessment of the use of them is rated as good in $58.9 \%, 66.1 \%$ and $72 \%$ of undergraduate students, graduate students and teachers respectively. The use of these tools is higher in undergraduate students $92.6 \%$ followed by graduate $82.6 \%$ and teachers $69.8 \%$. The knowledge of the various tools of generation and publication of content is present in the three groups. The recovery and finding information tool most used by teachers and graduate students were specialized databases. Conclusions: There is a positive trend to use these tools within the educational field. Keywords: Education, Higher educational technology, Web browser, Internet.
1. Departamento Académico de Estomatología Preventiva y Social

2. Departamento Académico de Estomatología Rehabilitadora

\section{Correspondencia}

Mg. Teresa Evaristo Chiyong

Facultad de Odontología. Universidad Nacional Mayor de San Marcos. Av. Germán Amézaga s/n. Lima 1, Perú.

Correo electrónico: t_evaristo2002@yahoo.es Silvia Chein: scheinv@unmsm.edu.pe Lita Ortiz: limaof09@gmail.com Lita Cáceres: litacaceres@ahoo.es Doris Salcedo: endodoncista@hotmail.com Marisa Jara: marisareima@hotmail.com Gerardo Ayala: gerardoayala17@yahoo.es 


\section{Introducción}

Las TIC de la web 2.0 considera el uso de aplicaciones y páginas web dispuestas a utilizar la inteligencia colectiva a través del software social, con el fin de facilitar los procesos de aprendizaje. De Harot ${ }^{1}$ clasifica estas herramientas según la función que desempeñan en: herramientas para generar y publicar contenidos, herramientas de recuperación de información y herramientas de comunicación

La web 2.0 o "web de las personas" se podría definir como un conjunto de tecnologías para la creación social de conocimiento, incorporando tres características esenciales: tecnología, conocimiento y usuarios; y se caracteriza por la creación colectiva de contenidos, el establecimiento de recursos compartidos y el control de la calidad de forma colaborativa entre los usuarios, también puede entenderse como facilitadora del cambio de paradigma en los procesos de aprendizaje. No se trata de un cambio tecnológico aislado, sino de un modelo constructivista, que entiende el aprendizaje como el resultado de la interacción y colaboración de las personas; y que sitúa al usuario, en este caso al estudiante, en el centro del proceso, con un papel activo en su propio aprendizaje. Según el ranking de las cien herramientas tecnológicas más utilizadas para el aprendizaje en el año 2008, según C4LPT Resource Centre, presentado por Esteve, ${ }^{2}$ se observa que la mayoría de estas son tecnologías 2.0 o colaborativas: Delicious, Google Reader, Google Docs, Skype, Moodle, Slideshare, Twitter, Ning, Youtube o Flickr, entre otras.

Nakano, ${ }^{3}$ en el 2011, encontró que el $70 \%$ de los estudiantes de una universidad de lima utiliza esta tecnología todos los días entre 3 y 4 horas diarias. Los mayores de 23 años se conectan a internet en mayor medida para trabajar o estudiar que para el ocio. Los estudiantes mayores de 23 años consumen internet para revisar contenidos, revistas, mientras que los mayores de 26 revisan bases de datos y revistas especializadas, portales educativos, páginas web generales, institucionales o temáticas.

Algieri $^{4}$ en el 2012, identificó que los espacios de enseñanza virtuales, con especificación de Moodle, constituyen una herramienta pedagógica que favorece la interacción docente-alumno. Los resultados permitieron concluir que el aporte de esta herramienta al proceso enseñanza aprendizaje es que desarrolla interacciones comunicativas a distancia, flexibi- lizando en tiempo y espacio el desarrollo de esta interacción, donde el docente se transforma en un "Tutor on line" que acompaña, guía y asesora. Este rol de tutor se centra en el estudiante, siendo el docente un facilitador del aprendizaje autónomo por parte de los alumnos y guía para la resolución de problemas planteados por el mismo.

A pesar de la fuerte evolución de las TIC, las prácticas docentes dominantes en las aulas no han cambiado en forma notoria. Existe un desfase entre la potencialidad de las TIC incorporadas en las aulas y la escasa renovación de los procesos de enseñanza aprendizaje. Las TIC se han ido incorporando en las universidades, a menudo asociadas a prácticas docentes poco participativas. Por ejemplo, en muchos casos simplemente se han sustituido las tradicionales pizarras por modernas presentaciones Power Point. Las tecnologías en sí no producen innovación educativa. Solo asociadas a adecuadas prácticas educativas pueden ser una gran fuente de posibilidades de aprendizaje contextualizado, por lo cual la presente investigación considera importante identificar el conocimiento, aplicación y la valoración de las TIC dentro del desarrollo académico. La finalidad del proceso formativo complementado con las TIC es lograr mejores resultados de aprendizaje, lo que se verá reflejado en la formación de ciudadanos competentes que puedan desenvolverse con las exigencias de esta nueva sociedad: la sociedad de la información y del conocimiento. La preocupación de las universidades, en especial de la Universidad Nacional Mayor de San Marcos, por ofrecer una enseńanza de calidad supone no solo brindar un cúmulo de conocimientos científicos y tecnológicos del área profesional, sino también conocer y considerar las variables implicadas en el proceso de enseńanza-aprendizaje dentro del avance y progreso de la sociedad. Y es en este contexto que la comunidad educativa con sus modos, medios y técnicas necesita adaptarse a una sociedad cada vez más apoyada en las tecnologías de la información y la comunicación (TIC), por lo que en el presente trabajo de investigación se tuvo como objetivo analizar el conocimiento, aplicación y apreciación sobre las tecnologías de la información y comunicación (TIC) de la web 2.0 en el ámbito educativo.

\section{Materiales y método}

- Tipo de estudio: Se desarrolló la investigación bajo un enfoque cuantitativo, de tipo descriptivo transversal y prospectivo.
- Población: Estudiantes regulares de pregrado y posgrado de la $\mathrm{Fa}$ cultad de odontología de la Universidad Nacional Mayor de San Marcos (UNMSM) que cursaron el año académico 2015 y docentes de pregrado y posgrado de dicha facultad en actividad académica durante el mismo año. Se excluyeron a estudiantes que llevaron menos de cursos durante el semestre académico.

- Muestra: Se desarrolló un muestreo probabilístico para estimación de una proporción y estratificando según año de estudios para contar con una representación de cada uno de los años de estudio de pregrado y de las respectivas especialidades en posgrado.Los estimadores utilizados fueron $\mathrm{p}=0.77$ (Hillca $\mathrm{N}^{5}$ ), precisión: 0.05 , nivel de confianza: $95 \%$. Quedando conformada la muestra por 173 estudiantes de pregrado, 109 de posgrado y 54 docentes.

- Procedimiento de recolección de datos: Se utilizó el método de encuesta a través de la técnica del cuestionario. Los recursos de la web 2.0 estudiados fueron los considerados por $\mathrm{Haro}^{2}$ y se clasificaron en tres: herramientas de comunicación, de generación y publicación de contenidos y de recuperación de información.

El cuestionario constó de 20 preguntas:

- Tres preguntas iniciales que permitieron identificar las formas de acceso al internet.

- Ocho preguntas correspondientes al conocimiento, aplicación y apreciación sobre las herramientas de comunicación.

- Seis preguntas correspondientes al conocimiento, aplicación y apreciación sobre las herramientas de generación y publicación de contenidos.

- Tres preguntas correspondientes al conocimiento, aplicación y apreciación sobre las herramientas de recuperación de información.

El cuestionario fue sometido a validación mediante juicio de experto, obteniéndose en cada una de las preguntas coeficientes V de Aiken iguales a 1. Se realizó el análisis de consistencia interna obteniéndose un coeficiente alfa de Conbrach de 0,75. 
Para la aplicación del cuestionario se coordinó con los docentes responsables de las asignaturas con el mayor número de estudiantes quienes brindaron un tiempo para el desarrollo del mismo, sin perjudicar el tiempo de descanso de los estudiantes. La selección de cada uno de los estudiantes fue realizada de manera aleatoria sistemática. La selección de los docentes fue realizada de manera aleatoria simple, a través de la relación de docentes de pregrado y posgrado proporcionada por la escuela académica y la unidad de posgrado respectivamente.

- Análisis de la información: El procesamiento de la información se realizó de manera computarizada haciendo uso del paquete estadístico SPSS versión 21. El análisis univariado se realizó a través de frecuencias absolutas y relativas, presentadas a través de tablas. El análisis bivariado se realizó a través de la prueba chi cuadrado de homogeneidad y de independencia. Se consideró un nivel de significancia del $5 \%$.

\section{Resultados}

- Herramientas de comunicación: Con respecto al conocimiento de estas herramientas, se observa que en los tres grupos es considerado principalmente como un mecanismo de compartir información, con porcentajes superiores al $85 \%$, entre los que están de acuerdo y totalmente de acuerdo. La mejora del vínculo docente y el desarrollo del trabajo colaborativo está por encima del $80 \%$ en los tres grupos, entre los que están de acuerdo y totalmente de acuerdo.

La apreciación sobre el uso de estas herramientas en el ámbito educativo es calificada como buena y excelente para los estudiantes de pre y posgrado; mientras que para los docentes es buena y regular. Estas diferencias son estadísticamente significativas.

En el ámbito académico estas herramientas son utilizadas con un porcentaje por encima del 69\%. Los docentes son los que menos las utilizan, y quienes más las uti- lizan son los estudiantes. La red social utilizada con mayor frecuencia tanto en el ámbito académico como no académico es el Facebook (Tabla 1).

- Herramientas de generación y publicación de contenidos: $\mathrm{El}$ conocimiento de los aplicativos de generación del conocimiento nivel de pregrado es acerca de Youtube, en un 79,9\%, Slideshare, 81,9\%, y en menor porcentaje Google Drive, 49,7 \%. La tendencia en este nivel es conocer las herramientas de generación de contenido en la mayoría de los casos. A nivel de posgrado los blogs, 65,7 \%, y Prezzi, $52,8 \%$, son los más reconocidos, tomando muy poco en cuenta a Wikipedia, 48,1 \%. A nivel de docentes, el reconocimiento de todas las herramientas mencionadas es mayor de $50 \%$ en cada caso. La percepción con respecto al uso de estas herramientas a nivel de pregrado y docentes fue buena, con porcentajes superiores al 50. Posgrado consideró bueno el $45 \%$, no

Tabla 1: Conocimiento ,apreciación y aplicación de las herramientas de comunicación en estudiantes y docentes de la Facultad de Odontología de la UNMSM. 2015

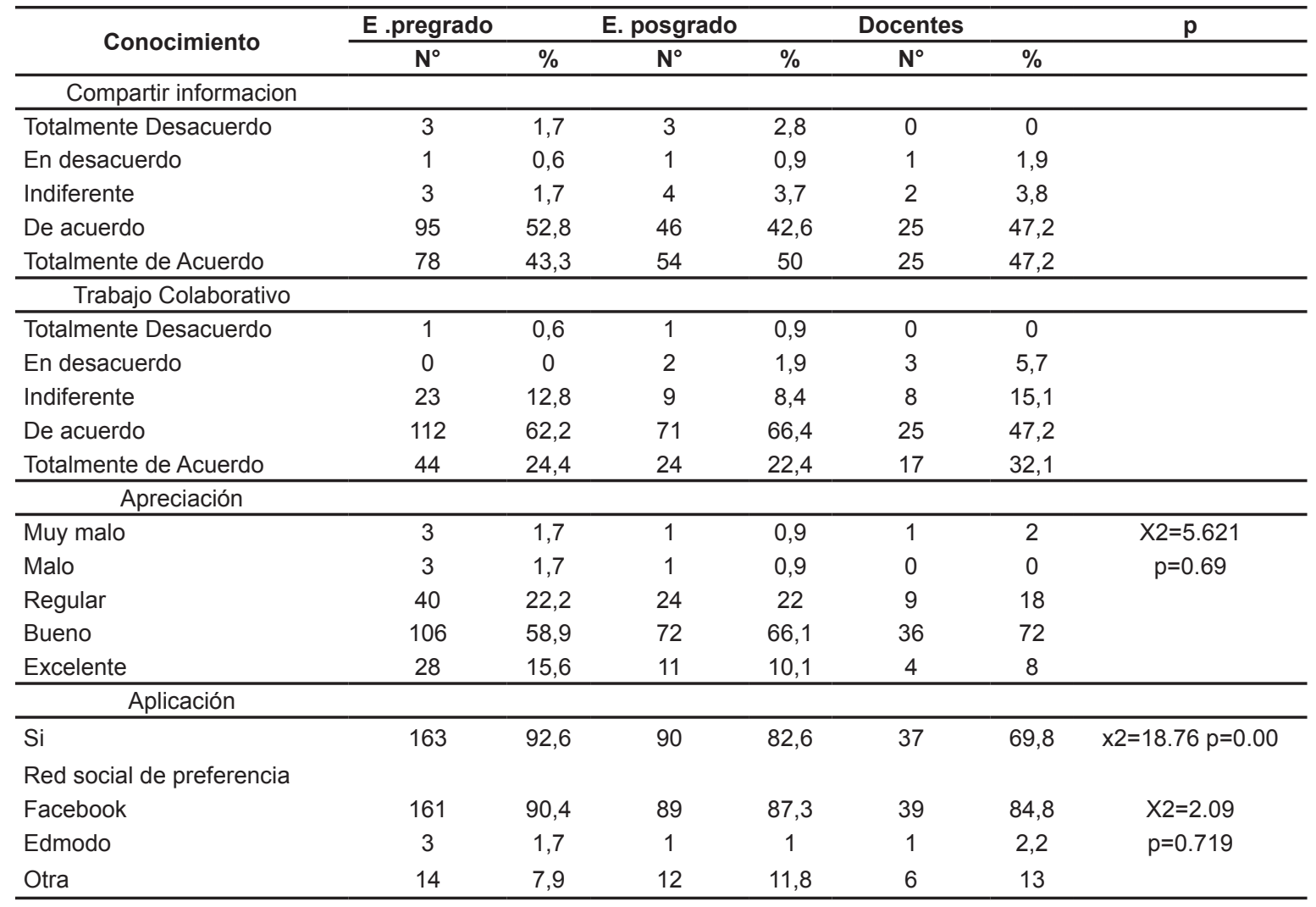


encontrándose diferencia significativa entre los grupos.

En el nivel de pregrado, el porcentaje de los que no utilizan estas herramientas es ligeramente mayor $(52,2 \%)$ que en posgrado y docentes, no encontrándose diferencias significativas en los resultados entre los grupos. p>0,05. La herramienta más utilizadas en pregrado es el blog, seguida del Prezzi, a diferencias del posgrado, donde se usa más el aplicativo Prezzi, seguido del Google Drive. A nivel de los docentes, al igual que los estudiantes de posgrado, es el Prezzi pero en este caso seguido de Youtube ( $\mathrm{Ta}$ bla 2).

- Herramientas de recuperación y búsqueda de información: La percepción sobre el uso de buscadores y metabuscadores es apreciado principalmente como buena, con porcentajes por encima del $53 \%$, siendo este valor mayor en docentes $(67,4 \%)$, no siendo estas diferencias estadísticamente significativas.
La herramienta de búsqueda utilizada por los estudiantes de pregrado fue en primer orden Google, seguida por la base de datos especializadas, mientras que en docentes y estudiantes de posgrado la base de datos especializada fue la utilizada en primer orden, seguida por Google (Tabla 3).

\section{Discusión}

Herramientas de comunicación: En esta investigación se observa que más del $92 \%$ considera a las herramientas de comunicación como herramientas para el uso educativo y concuerda con lo que Salinas ${ }^{6}$ propone. Las necesidades de la sociedad actual determina que las instituciones de educación superior deben flexibilizarse y desarrollar vías de integración de las tecnologías de la información y la comunicación en los procesos de formación, generando una nueva concepción de los alumnosusuarios, así como cambios de rol en los profesores y cambios administrativos en relación con los sistemas de comunicación y con el diseño y la distribución de la enseńanza.
Con respecto a la valoración de estas por los docentes y alumnos como herramienta interactiva, para desarrollar sus trabajos en equipo y difusión de actividades, concuerda con los resultados de Evaristo, ${ }^{7}$ que plantea que la tecnología de información y comunicación (TIC) brinda una serie de posibilidades para mejorar los procesos educativos, coincidiendo con Silva, ${ }^{8}$ quien plantea que actualmente existe consenso en que la implementación de los procesos innovadores de enseńanza potencian mejores aprendizajes.

Referente a la percepción de uso de estas herramientas, estos resultados concuerda con los resultados de Hui$1 \mathrm{lca}^{5}$ quien en un estudio desarrollado en estudiantes de Odontología de una universidad de Lima encontró que el mayor porcentaje de alumnos que usaron las TIC alcanzaron un rendimiento académico bueno $(56,3 \%) \mathrm{y}$, por ende, lo consideraron importante dentro de su proceso de aprendizaje.

Gutiérrez, ${ }^{9}$ a diferencia del presente estudio, encontró que solo un $20 \%$ de estudiantes tienen una visión optimista pro-TIC, considerándola como una

Tabla 2: Conocimiento ,apreciación y aplicación de las herramientas de generación y publicación de contenidos en estudiantes y docentes de la Facultad de Odontología de la UNMSM. 2015

\begin{tabular}{|c|c|c|c|c|c|c|c|}
\hline Conorimianto & E. pregrado & & E. posgrado & & Docentes & & $p$ \\
\hline conociminto & $\mathbf{N}^{\circ}$ & $\%$ & $\mathbf{N}^{\circ}$ & $\%$ & $\mathbf{N}^{\circ}$ & $\%$ & \\
\hline Prezzi(Si) & 94 & 54,7 & 57 & 52,8 & 30 & 61,2 & \\
\hline Blogs (Si) & 109 & 62,6 & 71 & 65,7 & 35 & 67,3 & \\
\hline Wikipedia (Si) & 131 & 74,4 & 52 & 48,1 & 30 & 60 & \\
\hline Google Drive(Si) & 87 & 49,7 & 60 & 55,6 & 29 & 55,8 & \\
\hline Youtube (Si) & 145 & 81,9 & 27 & 25 & 45 & 86,5 & \\
\hline Slideshare(Si) & 139 & 79,9 & 36 & 33,3 & 37 & 74 & \\
\hline \multicolumn{8}{|l|}{ Apreciación } \\
\hline Muy malo & 2 & 1,2 & 6 & 5,5 & 0 & 0 & $X 2=14.20$ \\
\hline Malo & 4 & 2,3 & 4 & 3,7 & 1 & 2,2 & $p=0.077$ \\
\hline Regular & 44 & 25,7 & 42 & 38,5 & 15 & 32,6 & \\
\hline Bueno & 102 & 59,6 & 49 & 45 & 26 & 56,5 & \\
\hline Excelente & 19 & 11,1 & 8 & 7,3 & 4 & 8,7 & \\
\hline \multicolumn{8}{|l|}{ Aplicación } \\
\hline $\mathrm{Si}$ & 85 & 47,8 & 62 & 56,9 & 34 & 63 & $x 2=4.778 p=0.09$ \\
\hline \multicolumn{8}{|l|}{ Herramientas utilizadas } \\
\hline Prezzi(Si) & 9 & 11,8 & 15 & 34,1 & 9 & 31 & $x 2=34.29$ \\
\hline Blogs (Si) & 34 & 44,7 & 1 & 2,3 & 4 & 13,8 & $p=0.000$ \\
\hline Wikipedia (Si) & 2 & 2,6 & 2 & 4,5 & 1 & 3,4 & \\
\hline Google Drive(Si) & 7 & 9,2 & 8 & 18,2 & 4 & 13,8 & \\
\hline Youtube (Si) & 19 & 25 & 11 & 25 & 6 & 20,7 & \\
\hline Slideshare(Si) & 5 & 6,6 & 7 & 15,9 & 5 & 17,2 & \\
\hline
\end{tabular}


Tabla 3: Apreciación y aplicación de las herramientas de recuperación y búsqueda de información en estudiantes y docentes de la Facultad de Odontología de la UNMSM. 2015

\begin{tabular}{|c|c|c|c|c|c|c|c|}
\hline Anrociación & E. pregrado & & E. posgrado & & Docentes & & p \\
\hline Aprecıacıon & $\mathbf{N}^{\circ}$ & $\%$ & $\mathbf{N}^{\circ}$ & $\%$ & $\mathbf{N}^{\circ}$ & $\%$ & \\
\hline Muy malo & 1 & 0,6 & 1 & 1 & 0 & 0 & $X 2=4.517$ \\
\hline Malo & 3 & 1,7 & 1 & 1 & 0 & 0 & $p=0.80$ \\
\hline Regular & 22 & 12,8 & 18 & 17,1 & 5 & 10,9 & \\
\hline Bueno & 105 & 61 & 56 & 53,3 & 31 & 67,4 & \\
\hline Excelente & 41 & 23,8 & 29 & 27,6 & 10 & 21,7 & \\
\hline Preferencia en orden de aplicación & $\overline{\mathbf{X}}$ & s & $\overline{\mathbf{X}}$ & $\mathbf{s}$ & $\overline{\mathbf{X}}$ & $\mathbf{s}$ & \\
\hline Google & 3,81 & 1,67 & 3,23 & 1,7 & 3,23 & 1,51 & \\
\hline Reader académico & 2,1 & 1,87 & 1,97 & 1,75 & 2,42 & 1,83 & \\
\hline Blogg lines & 1,31 & 1,58 & 1,11 & 1,58 & 1,22 & 1,49 & \\
\hline Freedly & 0,65 & 1,38 & 0,82 & 1,48 & 0,53 & 1,25 & \\
\hline Base de datos especializadas & 3,31 & 1,7 & 3,38 & 1,77 & 3,73 & 1,68 & \\
\hline
\end{tabular}

oportunidad para mejorar la comunicación. Una gran mayoría (75 \% de la muestra) se manifiesta o bien apáticos y desinteresados, o bien neutrales y pragmáticos, en el sentido de que, aunque las TIC no constituyen una parte significativa ni esencial de su vida, las aceptan en la medida que les sirvan para conseguir sus objetivos académicos más inmediatos.

Herramientas de generación y publicación de contenidos: La tendencia a nivel de pregrado en este nivel es conocer las herramientas de generación de contenido en la mayoría de los casos, siendo la principal reconocida Slideshare y en posgrado los blogs.

A nivel de docentes, el reconocimiento de todas las herramientas mencionadas es mayor de $50 \%$ en cada caso. Esto concuerda con el hecho de que autores como Cobo $^{10}$ y Muñoz ${ }^{11}$ demuestran la existencia de un proceso de selección natural en la web que permite a los sitios de buena calidad sobresalir y permanecer, mientras que los contenidos falsos o con poca calidad son los más propensos a desaparecer. Así tenemos el caso de Wikipedia, donde las entradas sin sentido o con poca calidad son eliminadas por los propios usuarios; o en caso de nunca ser buscadas, por los propios editores de la enciclopedia. Los aplicativos de las herramientas de generación de contenidos no están orientadas en este caso tanto al trabajo colaborativo sino, más bien, a compartir información y otras. Putland ${ }^{12}$ considera que estos aplicativos se orientan a la construcción de contenidos de forma colaborativa.

En la presente investigación a nivel de pregrado y docentes, se encontró bueno el uso de estos aplicativos, ubicándose en un porcentaje superior al 50. Posgrado consideró bueno el $45 \%$, no encontrándose diferencia significativa entre los grupos. Parte importante de estos resultados podría atribuirse a la falta de confianza que muchos profesores y alumnos demuestran todavía hacia un modelo más abierto de educación, donde los alumnos establecen y construyen aspectos cruciales del contenido de un curso. En el nivel de pregrado el porcentaje de los que no utilizan estas herramientas es ligeramente mayor (52,2\%). Posgrado y docentes presentan el mayor porcentaje en los que sí usan estas herramientas, no encontrándose diferencias significativas en los resultados entre los grupos. Sin embargo, el porcentaje de uso está por debajo del $65 \%$. Orante ${ }^{13}$ encontró insuficientes conocimientos y actitudes, en muchos casos negativas, de los estudiantes, comprobando que los alumnos de educación no están tan familiarizados con las TIC como se piensa; incluso como usuarios habituales de nuevas tecnologías ignoran su potencial didáctico y las posibles formas de integración en los currículos de la enseñanza obligatoria.

La herramientas más utilizada en pregrado es el blog. A nivel de los docentes, al igual que en los estudiantes de posgrado es el Prezzi. La diferencia significativa de los resultados entre los grupos puede deberse a la falta de conocimien- to de las mismas y de la ayuda que las nuevas herramientas y tecnologías de corte informático proporcionan en la organización y el flujo de información, dependiendo del comportamiento de las personas que acceden a ella.

Herramientas de recuperación y búsqueda de información: La percepción sobre el uso de buscadores y metabuscadores es apreciado principalmente como bueno, con porcentajes por encima del $53 \%$, siendo este valor mayor en docentes $(67,4 \%)$, no siendo estas diferencias estadísticamente significativas. Baelo ${ }^{14}$ determinó que los recursos relacionados con la búsqueda de información en la red Internet fue la segunda herramienta más utilizada por los docentes.

La herramienta de búsqueda utilizada por los estudiantes de pregrado fue en primer orden Google, seguida por la base de datos especializadas, mientras que en docentes y estudiantes de posgrado la base de datos especializada fue la utilizada en primer orden, seguida por Google.

\section{Conclusión}

Las tecnologías de la información y comunicación (TIC) de la web 2.0 son consideradas útiles para el desarrollo de los procesos académico, pudiendo mejorarse la aplicación dentro de los mismos.

\section{Agradecimiento}

Al Vicerrectorado de Investigación por el financiamiento económico para el desarrollo del presente estudio. 


\section{Referencias}

1. García A L .Bases, mediaciones y futuro de la educación a distancia en la sociedad digital. 2014. Editorial Sintesis. Madrid. 318p.

2. Esteve F. Bolonia y las Tic de la docencia 1.0 al aprendizaje 2.0. La Cuestion Universitaria. 2009. (5). Pp.59-68. [Acceso 1 Diciembre de 2014]; Disponible en http://www. anobium.es/docs/gc_fichas/doc/ LRSPFDzlbc.pdf

3. Nakano $T$, Azabache $H$, De la Flor M. Evaristo I, Molla I, Morla K, Valdez N. Uso, acceso y apreciaciones de las tecnologías de la información y comunicaciones es estudiantes de la Pontificia Universidad Católica del Perú. 2011. Grupo Avatar. PUCP.

4. Algieri, D; Dogliotti, C; Rey, L; Mazzoglio y Nabar, M; Gazzotti, A; Pró, E. Interacción docentealumno mediante la utilización de sistema virtual .Hosp. Aeronáut. Cent 2012. 7(1): 21-25. . [Acceso 02 de Diciembre 2014]; Disponible en: http://www.hac. mil.ar/publicaciones/revismedic/0701/21-25.pdf

5. Huillca-Castillo N, Ávalos-Márquez J. Las tecnologías de la información y comunicación y su relación con el rendimiento acadé- mico en estudiantes de Odontología. Kiru 2013;10(1):20-24.

6. Salinas, Jesús. Innovación docente y uso de las TIC en la enseñanza universitaria. Revista de Universidad y Sociedad del Conocimiento, 2004. 1 ( 1), pp. 1-16

7. Evaristo I, La TIC más allá del aula: El reto del Grupo Avatar. En Blanco \& Negro 2012: 3(2). [Acceso 15 de Noviembre 2014]; Disponible http://revistas.pucp.edu.pe/ index.php/enblancoynegro/article/ view/3859

8. Silva J. Formación docente en un espacio virtual de aprendizaje: una experiencia concreta en el contexto chileno. Teoría de la Educación. Educación y Cultura en la Sociedad de la Información [Revista en Internet].2006. [Acceso 15 de Noviembre de 2014]; 7(1) Disponible en:http://www.redalyc.org/articulo.oa?id $=201021084009$.

9. Gutiérrez-Marian A, Palacios-Picos A, Torres-Egido L. Tribus digitales en las aulas universitarias., Revista Científica de Educomunicación. Comunicar. 2010.17(34).173-181

10. Cobo R, C; Pardo K H. 2007.Planeta Web 2.0. Inteligencia colectiva o medios fast food. 2007. Grup de Recerca d'Interaccions Digitals, Universitat de Vic..Flacso México. Barcelona .158p. . [Acceso 5
Noviembre de 2014]; Disponible en: file:///C:/Users/DELL/Downloads/planeta_web2.pdf

11. Muñoz-Cano J, Córdova J, Priego $H$. Dificultades y facilidades para el desarrollo de un proceso de innovación educativa con base en las Tecnologías de la Información y Comunicación (TIC). Formación Universitaria .2012 .5 (1) . [Acceso 02 Diciembre de 2014]; Disponible en: http://www.scielo.cl/pdf/ formuniv/v5n1/art02.pdf

12. Putland .Blogs, Wikis, RSS and there's more? Web 2.0 on the march. Education.au.1 de septiembre, 2006. Disponible en:http://www.educationau.edu. au/jahia/Jahia/home/pid/337

13. Baelo R, Canton I. Las TIC en las Universidades de Castilla y León. Revista Científica de Educomunicación. Comunicar. 2010.18(35).159-166

14. Orantes S.L. Actitudes, dominio y uso de las tecnologías de la información y la comunicación (TIC) de los docentes de las universidades privadas de El Salvador. Informe de la Universidad Tecnológica de El Salvador.2009.Disponible en: http://www.utec.edu.sv/me$\mathrm{dia} /$ investigaciones/files/ActitudesdominioyusodelasTICdelosdocentes.PorLui.pdf. 